\title{
Approximate Scheduling of DERs with Discrete Complex Injections
}

\author{
Sanmukh Kuppannagari \\ kuppanna@usc.edu \\ Ming Hsieh Department of Electrical \\ and Computer Engineering, \\ University of Southern California \\ Los Angeles, California
}

\author{
Rajgopal Kannan \\ rajgopal.kannan.civ@mail.mil \\ US Army Research Lab, 12015 \\ Waterfront Drive \\ Playa Vista, California
}

\author{
Viktor K. Prasanna \\ prasanna@usc.edu \\ Ming Hsieh Department of Electrical \\ and Computer Engineering, \\ University of Southern California \\ Los Angeles, California
}

\begin{abstract}
Rapid penetration of renewable energy based Distributed Energy Resources (DER) has the potential to exacerbate the challenges inherent in grid frequency and voltage regulation. However, their real time controllability can be leveraged to not only mitigate such challenges, but improve the economics and quality of grid operations. A plethora of DER scheduling algorithms have been developed for fast and efficient scheduling of these resources. These algorithms schedule the complex - real and reactive power injected into (or drawn from) the grid by the DERs under various cost objective functions and grid constraints. However, a vast majority of such algorithms assume the feasible range of power injections to be continuous. In reality, the control options available for several DERs form a discrete space. This implies that the feasible range of power injections also forms a discrete space. This makes DER scheduling an NP-hard problem. Integer/Mixed Integer program based algorithms, which guarantee optimality in the objective value, have been developed. But these are prohibitively expensive in terms of computational time requirements. On the other hand, fast heuristic based solutions have also been developed. But as they do not provide any guarantee on the optimality of the objective, or on satisfying the constraints, they are unreliable for grid operations. Recent works have developed approximation algorithms which offer the best of both the approaches above: (near) optimality and low computational complexity, both of which are governed by a user defined accuracy parameter $\epsilon$. In this work, we significantly improve upon such existing works by developing an approximation algorithm which schedules DERs with complex injections (as opposed to only real injection) and whose runtime is polynomial (as opposed to exponential) in $\frac{1}{\epsilon}$. We provide theoretical analysis and support them with experimental results to validate the guarantees provided by our approximation algorithm.
\end{abstract}

\section{CCS CONCEPTS}

- Hardware $\rightarrow$ Smart grid; • Theory of computation $\rightarrow$ Packing and covering problems.

Permission to make digital or hard copies of all or part of this work for personal or classroom use is granted without fee provided that copies are not made or distributed for profit or commercial advantage and that copies bear this notice and the full citation on the first page. Copyrights for components of this work owned by others than ACM must be honored. Abstracting with credit is permitted. To copy otherwise, or republish, to post on servers or to redistribute to lists, requires prior specific permission and/or a fee. Request permissions from permissions@acm.org.

e-Energy '19, June 25-28, 2019, Phoenix, AZ, USA

(C) 2019 Association for Computing Machinery.

ACM ISBN 978-1-4503-6671-7/19/06 . \$ \$15.00

https://doi.org/10.1145/3307772.3328311

\section{KEYWORDS}

DER Scheduling, Discrete Complex Injection, Approximation Algorithm

ACM Reference Format:

Sanmukh Kuppannagari, Rajgopal Kannan, and Viktor K. Prasanna. 2019. Approximate Scheduling of DERs with Discrete Complex Injections. In Proceedings of the Tenth ACM International Conference on Future Energy Systems (e-Energy '19), June 25-28, 2019, Phoenix, AZ, USA. ACM, New York, NY, USA, 11 pages. https://doi.org/10.1145/3307772.3328311

\section{INTRODUCTION}

Driven by the renewable energy portfolio mandates adopted by several governments such as Hawaii [3], California [4], European Union [35], etc., penetration of renewable energy based Distributed Energy Resources (DERs) is increasing rapidly [15]. However, this increase can potentially escalate the complexity of two of the most critical grid operations: frequency regulation and voltage regulation. Renewable energy output is largely governed by weather conditions - which are highly variable. This leads to frequent supply-demand mismatches, thereby causing a drift in frequency from the standard value $(50 / 60 \mathrm{~Hz})$ [28]. Similarly, the increase in power injected by DERs into the distribution grid leads to an increase in voltages on the feeders which needs regulating [28]. The severity of these challenges is evident from the conservative limits (15\% [2]) on renewable penetration levels in the distribution grid adopted by certain utilities.

However, the technological advances in DERs provide us with opportunities to address the challenges mentioned above. DERs allow real time controllability through protocols such as Active Network Management (ANM) [29]. Moreover, the low ramp times of smart inverters [37] (devices which convert DC output of certain DERs to AC output to inject into the grid) allows DERs to respond quickly and provide needed support for grid operations. Thus, DERs can be used to not only mitigate challenges in grid operations, but to also lower the cost of grid operations [31] and improve the quality of power delivery under abnormal conditions [7].

To enable this, numerous DER scheduling algorithms have been developed in the literature (Section 2). Algorithms which are theoretically optimal and have a low runtime complexity have been developed assuming the feasible range of power injections forms a continuous space i.e. these algorithms assume that each DER can inject any value between a minimum and a maximum value. However, in reality, several DERs such as HVAC systems [16], EVs [18], PV systems [12] etc. exhibit discrete feasible range. Such DERs have a fixed set of control options and selection of a certain control option 
at a given time injects a fixed value into the grid. The number of possible injection values is equal to the number of possible control options, thus, forming a discrete feasible space.

DER scheduling with discrete feasible space is NP-hard [20, 24]. Thus, no optimal algorithm with polynomial runtime complexity can exist. Until recently, works focusing on discrete feasible space fell into one of the following two categories: 1) Integer/Mixed Integer Program based algorithms which are computationally expensive, and 2) Heuristic solutions with no theoretical guarantees. The former is suitable for offline scheduling and is highly susceptible to prediction errors inherent in renewable energy generation prediction. The latter solutions suffer from uncertainty in terms of satisfying grid constraints and optimality criteria and in the worst case can have constraint/criteria violations which are unbounded. This makes them unreliable for critical grid operations.

Approximation algorithms for scheduling DERs have been developed recently to address these challenges. As NP-hard problems cannot have optimal polynomial runtime complexity solutions as per our current knowledge, approximation algorithms ensure polynomial runtime complexity by violating the constraints/criteria. However, the worst case violation is bounded by a user defined accuracy parameter which also governs the runtime. Thus, the user can obtain a solution which is close to optimal or satisfies constraints within some threshold, where the threshold can be determined by the user based on available computational resources.

In this work, we significantly improve upon the existing approximate DER scheduling algorithms. Our algorithm improves the runtime complexity from exponential in $\frac{1}{\epsilon}$ and $T$ in $[18,20]$ to polynomial in $\frac{1}{\epsilon}$ and $T^{1}$. Here, $\epsilon>0$ is the user defined accuracy parameter and $T$ is the number of intervals in the scheduling horizon. Additionally, we consider finer grained constraints on real and reactive power injections individually, as opposed to a combined apparent power constraint as proposed by the authors in $[18,20]$. They propose an LP rounding based approximation that schedules both active $P_{i}$ and reactive power $Q_{i}$ injections for each DER $i$, however the total power injected is constrained by an apparent power constraint $W=\sqrt{\left(\sum_{i} P_{i}\right)^{2}+\left(\sum_{i} Q_{i}\right)^{2}}$. This is a significant limitation since a limit on apparent power is sufficient for enforcing thermal capacity constraints but enforcing finer grained constraints as needed by modern devices (such as power factor constraints in PV inverters) requires individual constraints on real and reactive power. The simplicity of our solution makes it extensible, for example to enforce apparent power constraints as in [20], along with individual real and reactive power constraints, without losing theoretical guarantees. Our algorithm is more sophisticated that the algorithm proposed in [24] which performs net-load balancing by curtailment of real power of load and generation and cannot be easily extended for scheduling DERs with complex injections. We provide rigorous theoretical proofs and support them with experimental results to illustrate the effectiveness of our algorithms. Additionally, we provide real world applications and illustrate the extensibility of our algorithm by defining more complicated problems which use our algorithm as a sub-routine.

The major contributions of this work are as follows:

\footnotetext{
${ }^{1}$ The algorithm in $[18,20]$ targets a more complex setting. Please see Section 5 for details
}

- We develop an approximation algorithm for scheduling DERs which obtains the minimum cost solution in each interval, while ensuring that the difference between the targeted and achieved power (real and reactive) is within a factor of $(1+\epsilon)$, where $\epsilon$ is an accuracy parameter. Furthermore, our algorithm improves the runtime complexity of state-of-the-art algorithm $[18,20]$ from exponential in $\frac{1}{\epsilon}, T$ to polynomial in $\frac{1}{\epsilon}, T^{2}$.

- We generalize the apparent power constraint $[18,20]$ on real and reactive power (i.e. the limit on sum of squares of real plus reactive power) by considering finer grained constraints on real and reactive power injections individually. Moreover, we show how the apparent power constraint can trivially be implemented using our algorithm.

- We demonstrate the applicability of our algorithm by proposing a novel application: real and reactive support using smart inverters from large power plants in response to broader grid conditions. We also show how the traditional problem of PV/DER curtailment can be solved trivially using our algorithm.

\section{RELATED WORKS}

Significant research has been done on scheduling of DERs such as Demand Response (flexible load), energy storage systems, PV systems, EVs, etc. For Demand Response, we refer the readers to the survey paper [36] which thoroughly summarizes the research in this area. For EV systems, the survey paper [38] does the same. As evident from these surveys, DER scheduling assuming continuous feasible range has received significant attention using convex optimization techniques. However, for the discrete scenario, the focus has been on developing Integer/Mixed Integer program or heuristic based solutions which as mentioned in the previous section have their limitations.

The surveys mentioned above do not list the research done in the development of approximation algorithms. Previously, approximation algorithms were developed to achieve peak reduction. Peak reduction is a specific case of DER scheduling where the DER, in this case flexible load, is scheduled such that the peak load during the decision horizon is minimized. Several constant factor algorithms based on strip packing have been developed for the same $[6,30,34,40]$.

Generalized approximation algorithms for DER scheduling have only been developed recently. Works such as [24-26] developed approximation algorithms assuming the DERs output only real value. While true for traditional inverters, modern smart inverters support injection of both real and reactive values from the DERs. This limits the applicability of such algorithms. Authors in $[9,18,20]$ developed a PTAS: Polynomial Time Approximation Scheme. PTAS, while polynomial in the input size are still exponential in $\frac{1}{\epsilon}$, where $\epsilon$ is a user defined accuracy parameter. For example, for $N$ DERs, and $\epsilon=0.1$ i.e. $10 \%$ accuracy, the runtime is $O\left(N^{\frac{1}{\epsilon}}\right)=O\left(N^{10}\right)$ which is exorbitant. Moreover, their formulation has a constraint limiting the apparent power. This can lead to solutions with undesired effect on power factor (for example very low power factor in distribution feeders which are not allowed as per standards [8])

\footnotetext{
${ }^{2}$ See footnote 1
} 
while still satisfying the apparent power constraint. Also, note that their algorithm is exponential in $T$, the number of intervals.

We address these issues by developing an FPTAS: Fully Polynomial Time Approximation Scheme for scheduling DERs with real and reactive output. The runtime complexity of our algorithm is polynomial both in the input size, including $T$ - the number of time intervals in the scheduling horizon and the accuracy parameter $\frac{1}{\epsilon}$.

\section{SCHEDULING DERS WITH DISCRETE COMPLEX INJECTIONS}

\subsection{Model}

A power distribution grid consists of various components such as sub-station, transformers, loads, Distributed Energy Resources, voltage regulators etc. In this work, we only focus on scheduling the DERs installed in the power grid.

3.1.1 Smart Grid Model. We assume that there are $N$ controllable DERs. Each DER is associated with $M$ strategies. We assume the presence of a centralized controller which sends remote commands to each DER to switch it into a desired strategy. The decision needs to be taken for each interval $t$ of a scheduling horizon $T$. For simplicity of discussion here, we consider scheduling for a single interval $t$ and drop the notation $t$. In Section B in the appendix, we show how scheduling can be performed over a horizon with constraints creating inter dependencies among the intervals.

3.1.2 DER Strategies. We use the notation $[a]$ to denote the set of integers 1 through $a$ i.e. $\{1,2, \ldots, a\}$. We assume that each DER $i \in[N]$ is associated with a set of strategies $s_{i j}=\left(\gamma_{\mathbf{i j}}, c_{i j}\right) \forall j \in$ $[M]$. Here the number of strategies $M$ is without loss of generality as DERs which have a smaller number of strategies can set the remaining strategies to be some default strategy. $\gamma_{\mathrm{ij}}=\left(p_{i j}, q_{i j}\right)$ is a 2-dimensional vector denoting the real and reactive output power corresponding to strategy $j$ (of DER $i$ ). $c_{i j}$ is the corresponding cost. For example, if the DER is a PV with smart inverter, $\gamma_{\mathrm{ij}}$ could correspond to setpoints i.e. power factor settings. A DER can follow exactly one strategy in any given interval. We only consider the absolute values of $p_{i j}$ and $q_{i j}$. This implies that our algorithm is applicable in the scenarios where $\forall i, j p_{i j}\left(q_{i j}\right) \leq 0$ or $p_{i j}\left(q_{i j}\right) \geq 0$.

3.1.3 Decision Variables. We use the $0-1$ matrix $X \in R^{N \times M}$ to denote a single decision made by the controller. Here, $X_{i j}=1$ implies that DER $i$ should adopt strategy $j$, otherwise $X_{i j}$ is set to 0 . Let $\mathcal{X}$ denote the space of all possible valid decisions. $X \in \mathcal{X}$ is valid if $\forall i: \sum_{j \in[M]} X_{i j}=1$.

3.1.4 Objective. Our objective is to find the minimal cost decision matrix $X$, i.e $\operatorname{argmin}_{X} C_{X}$, where $C_{X}=\sum_{i \in[N]} \sum_{j \in[M]} c_{i j} X_{i j}$.

3.1.5 Constraints. In addition to restricting the decisions to be in the feasible region $\mathcal{X}$, the constraints of our algorithmic framework are the achievement of real and reactive target values $P$ and $Q$. We use the notation $\Gamma=(P, Q)$ to denote the target value. A decision $X \in X$ is said to satisfy the constraints if $P-\sum_{i \in[N]} \sum_{j \in[M]} p_{i j} X_{i j} \leq$ $\delta$ and $Q-\sum_{i \in[N]} \sum_{j \in[M]} q_{i j} X_{i j} \leq \delta$ i.e. the decision $X$ achieved a real and reactive value in the range $P-\delta: P$ and $Q-\delta: Q$ where $\delta$ is a threshold value.

\begin{tabular}{|l|l|}
\hline Notation & Meaning \\
\hline$N$ & Number of DERs \\
\hline$M$ & Number of strategies available for each DER \\
\hline$\gamma_{i j}=\left(p_{i j}, q_{i j}\right)$ & $\begin{array}{l}\text { (Real, Reactive) power injection of DER } i \text { adopt- } \\
\text { ing strategy } j\end{array}$ \\
\hline$c_{i j}$ & cost of DER $i$ adopting strategy $j$ \\
\hline$X$ & $0-1$ decision matrix \\
\hline$X_{i j}$ & $\begin{array}{l}0-1 \text { decision variable corresponding to DER } i \\
\text { adopting strategy } j\end{array}$ \\
\hline$\Gamma=(P, Q)$ & (Real, Reactive) power target \\
\hline$\Gamma_{a}=\left(P_{a}, Q_{a}\right)$ & (Real, Reactive) power achieved by a solution \\
\hline$P_{\max }, Q_{\max }$ & $\begin{array}{l}\text { Maximum bound on real and reactive value that } \\
\text { can be achieved using all DERs }\end{array}$ \\
\hline$P_{\min }, Q_{\min }$ & $\begin{array}{l}\text { Minimum non-zero real and reactive value that } \\
\text { can be achieved using any DER }\end{array}$ \\
\hline$\epsilon$ & Accuracy parameter \\
\hline$p f$ & Power Factor \\
\hline$W$ & Apparent power target \\
\hline$[a]$ & Denotes the set of integers $\{1,2, \ldots, a\}$ \\
\hline$\Phi$ & $\begin{array}{l}\text { Dynamic Programming recurrence relation and } \\
\text { table }\end{array}$ \\
\hline
\end{tabular}

Table 1: Notations used in this paper

In Section 4, we show how this very simple method of modeling the constraints can be used to model more sophisticated constraints such as real and reactive power thresholds or limits on apparent power, as required by specific applications. We summarize the notations used in this work in Table 1.

\subsection{Problem Definition and Hardness}

3.2.1 Problem Definition. Using the model defined in Section 3.1, the problem of minimum cost scheduling of DERs with discrete complex injections can be formulated using the following Integer Program:

$$
\min _{X \in \mathcal{X}} C_{X}
$$

subject to:

$$
\begin{aligned}
& P-\sum_{i \in[N]} \sum_{j \in[M]} p_{i j} X_{i j} \leq \delta \\
& Q-\sum_{i \in[N]} \sum_{j \in[M]} q_{i j} X_{i j} \leq \delta \\
& X_{i j} \in\{0,1\}
\end{aligned}
$$

Equation 1a minimizes the cost over all decision variables $X \in \mathcal{X}$, Equations $1 \mathrm{~b}$ and $1 \mathrm{c}$ ensure that the achieved real and reactive targets are within some $\delta$ value of the target values $P$ and $Q$. In other words, the problem tries to find the minimum cost solution within the range $P-\delta: P, Q-\delta: Q$. Equation $1 \mathrm{~d}$ denotes the constraint that the decision variables are $0-1$ integers. This constraint is responsible for the discrete nature of the problem. 


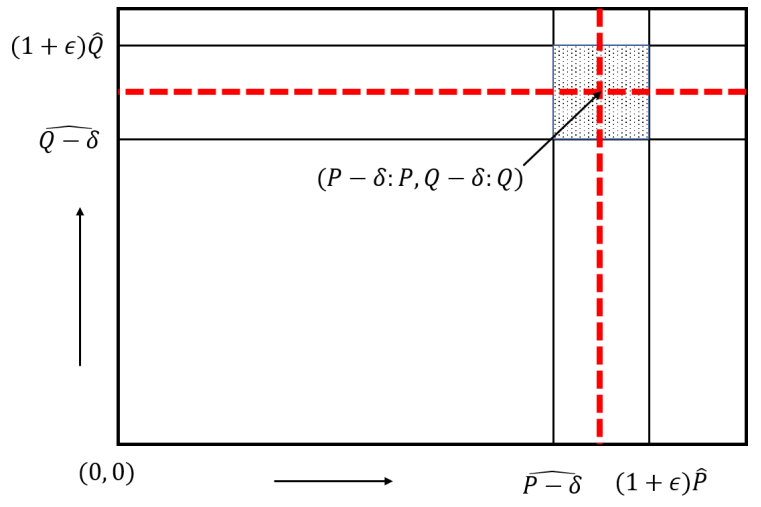

Figure 1: Instead of selecting in the range $P-\delta: P$ and $Q-\delta$ : $Q$, which is computationally expensive, Approx-DER selects the minimum cost solution in the range $\widehat{P-\delta}:(1+\epsilon) \widehat{P}$ and $\widehat{Q-\delta}:(1+\epsilon) \widehat{Q}$ (shaded portion)

3.2.2 Hardness of Approximability. It is evident that the problem defined by Equations 1a-1d is similar to the multi-constraint (multidimensional) knapsack problem [13] with two constraints (the difference due to $\delta$ is inconsequential in this case). It has been shown in [39] that multi-constraint knapsack (2-dimensional knapsack) cannot have a solution better than a PTAS, unless $P==N P$ i.e. unless $P==N P$ there cannot be a solution which is polynomial in both input size and $\frac{1}{\epsilon}$ while the constraints are satisfied and the objective is violated by a factor of $(1 \pm O(\epsilon))$. Heuristic solutions such as creating an off line table to store the strategies will not work due to the following reasons: (a) the size of the table will grow exponentially, and (b) even though the algorithm does not consider prediction uncertainty, the output of each control knob varies significantly due to the effect of weather conditions thereby making the creation of any off line table infeasible.

To work around this result and obtain a solution polynomial in $\frac{1}{\epsilon}$, we allow the constraints to be violated by at most a factor of $1+\epsilon$. Thus, we obtain a minimum cost solution which is within $1+\epsilon$ factor of the targeted $P$ and $Q$ values.

\subsection{Approx-DER Scheduling}

We develop an algorithm: Approx-DER for the problem defined by equations $1 \mathrm{a}-1 \mathrm{~d}$. The basic idea of the algorithm is as follows: We assume that the DERs are ordered as $[N]$. We assume that possible output values of the DERs are integers. These assumptions can be obtained by appropriate scaling. We create a dynamic programming recurrence relation which takes a node $i \in[N]$ and $\gamma=(p, q) \mid p \in[P], q \in[Q]$, as input and outputs the minimum cost needed to achieve $\gamma$ using nodes 1 through $i$. For $i=N$ and $\gamma=\Gamma$, we obtain the minimum cost solution. The actual strategies can be recovered by traversing the dynamic programming table. For simplicity of discussion, we will omit $\delta$ in the notations. We will implicitly assume that a dynamic programming traversal to obtain minimum cost solution for target $\Gamma=(P, Q)$ will output the minimum cost solution in the range: $(P-\delta: P, Q-\delta: Q)$.

However, the size and runtime of the algorithm is governed by $\Gamma$ which can be very large. Hence, we use a rounding approximation to reduce them. As shown in Figure 1, given an accuracy parameter $\epsilon$, we output a solution which is of the minimum cost and is in the vicinity of the target i.e. the achieved value $\frac{\Gamma}{(1+\epsilon)}<\Gamma_{a}<(1+\epsilon) \Gamma$.

Let $\Gamma_{\max }=\left(P_{\max }, Q_{\max }\right)$ be the bound on the maximum possible output that can be achieved collectively by all the DERs. Also, let $\Gamma_{\text {min }}=\left(P_{\text {min }}, Q_{\text {min }}\right)$ be the minimum possible such value greater than zero. We define the following partitions:

$$
\begin{array}{r}
\left\{[0],\left[P_{\min }, P_{\min }(1+\epsilon)^{\frac{1}{N}}\right),\left[P_{\min }(1+\epsilon)^{\frac{1}{N}}, P_{\min }(1+\epsilon)^{\frac{2}{N}}\right), \ldots,\right. \\
\left.\left[P_{\min }(1+\epsilon)^{\frac{k p-1}{N}}, P_{\min }(1+\epsilon)^{\frac{k p}{N}}\right)\right\} \\
\left\{[0],\left[Q_{\min }, Q_{\min }(1+\epsilon)^{\frac{1}{N}}\right),\left[Q_{\min }(1+\epsilon)^{\frac{1}{N}}, Q_{\min }(1+\epsilon)^{\frac{2}{N}}\right), \ldots,\right. \\
\left.\left[Q_{\min }(1+\epsilon)^{\frac{k_{q}-1}{N}}, Q_{\min }(1+\epsilon)^{\frac{k q}{N}}\right)\right\}
\end{array}
$$

where $k_{p}=\left\lceil N \log _{1+\epsilon}\left(\frac{P_{\max }}{P_{\min }}\right)\right\rceil$ and $k_{q}=\left\lceil N \log _{1+\epsilon}\left(\frac{Q_{\max }}{Q_{\min }}\right)\right\rceil$. We use the lowest value of a partition to represent it. We use $\widehat{p} \in$ $\left\{0, P_{\min }, \ldots, P_{\min }(1+\epsilon)^{\frac{k_{p}-1}{N}}\right\}$ and $\widehat{q} \in\left\{0, Q_{\min }, \ldots, Q_{\min }(1+\right.$ $\left.\epsilon)^{\frac{k_{q}-1}{N}}\right\}$ to index the partitions. We say that a value $p \in \widehat{p}$ if $p$ falls in the partition indexed by $\widehat{p}$. We use the corresponding notation for $q$.

We now define the dynamic programming function $\Phi(\widehat{p}, \widehat{q}, i)$ which denotes the minimum cost of achieving $\widehat{p}, \widehat{q}$ using DERs 1 through $i$. As defined before, $s_{i j}=\left(p_{i j}, q_{i j}, c_{i j}\right)$ is DER $i$ 's strategy $j$. The recurrence relation is defined as follows:

$$
\Phi(\widehat{p}, \widehat{q}, i)=\left\{\begin{array}{c}
\min _{j \in[N]} c_{i j} \text { if } i=1 \text { and } \exists s_{1 j} \mid p_{1 j} \in \widehat{p}, q_{1 j} \in \widehat{q} \\
\min _{\widehat{p}^{\prime}, \widehat{q}^{\prime}} \Phi\left(\widehat{p}^{\prime}, \widehat{q}^{\prime}, i-1\right)+c_{i j} \\
\quad \text { if } \exists s_{i j} \mid \widehat{p}^{\prime}+p_{i j} \in \widehat{p} \text { and } \widehat{q}^{\prime}+q_{i j} \in \widehat{q}
\end{array}\right.
$$

We also use the notation $\Phi$ to denote the dynamic programming table. $\Phi$ is initialized will all values set to $\infty$. Now, once $\Phi$ is filled, the minimum cost entry from $\Phi(\widehat{P-\delta}:(1+\epsilon) \widehat{P}, \widehat{Q-\delta}:(1+\epsilon) \widehat{Q}, N)$ denotes the desired solution. $\Phi$ can be traversed backwards to obtain the DER strategies. If all entries $\Phi(\widehat{P-\delta}:(1+\epsilon) \widehat{P}, \widehat{Q-\delta}:(1+$ $\epsilon) \widehat{Q}, N)=\infty$, no solution exists. For a maximization problem, we can replace $\infty$ with $-\infty$.

Therefore, given $\Gamma=(P, Q)$ and an accuracy parameter $\epsilon$, we have the following theorem:

THEOREm 3.1. Approx-DER is a polynomial time algorithm for scheduling DERs with discrete complex injections which outputs a minimum cost solution and which in the worst case violates the targeted real and reactive values by a factor of $(1+\epsilon)$.

A detailed proof of the theorem is presented in the appendix in Section A. Additionally, the algorithm to perform approximate DER scheduling over a horizon consisting of multiple time intervals is discussed in the appendix in Section B.

\section{APPLICATIONS}

The simplicity of our Approx DER scheduling algorithm allows its application to support a variety of objective functions and constrains. In this section, we provide a few examples of real world applications that can be implemented using approximate DER scheduling algorithm. As noted before, we will omit $\delta$ for simplicity of 


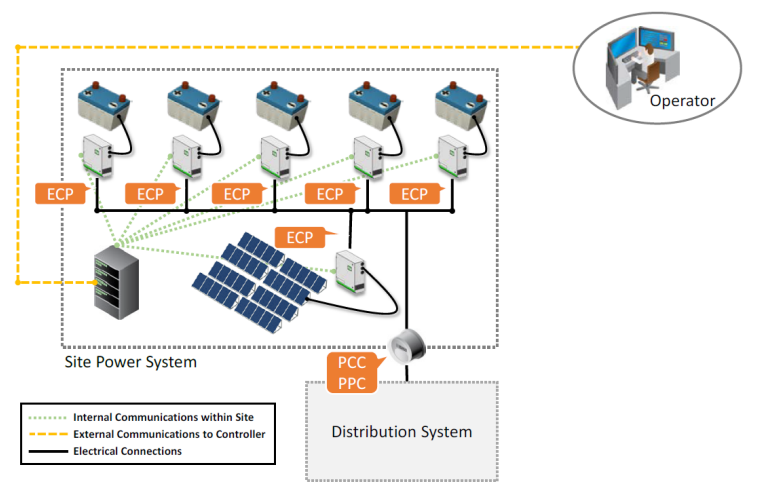

Figure 2: A large plant consisting of DERs connected to Smart Inverters. Figure borrowed from [1]. Here ECP stands for Electrical Connection Point, PCC stands for Point of Common Coupling and PPC stands for Point of Plant Control

presentation and will assume that the algorithm will search the appropriate range to find a minimum cost solution.

\subsection{Real and reactive support using Smart Inverters}

We consider the problem of scheduling DERs in a large power plant to provide real and reactive power support to the distribution grid. We assume that each DER is connected to a smart inverter. A high level overview of such a plant is shown in Figure 2.

We first briefly describe how a DER connected to a smart inverter functions. DERs such as storage, PV etc. output DC power at any given instant. Let the DC power output by DER $i$ be $W_{i}$. A smart inverter converts this DC power into sinusoidal AC complex (real and reactive) power and injects into the grid. A smart inverter is configured using a setting called power factor which determines the amount of real $P_{i}$ and reactive $Q_{i}$ power to be injected into the grid, for DER $i$ given $W_{i}$. The following equations govern the injection:

$$
\begin{array}{r}
p f=\frac{P_{i}}{W_{i}} \\
W_{i}=\sqrt{P_{i}^{2}+Q_{i}^{2}}
\end{array}
$$

where $p f$ is the power factor. Therefore, in this scenario, the control options for each DER $i$ are determined by the available power factor settings denoted as $p f_{i j} \forall j \in[M]$, where $M$ is the number of available power factor settings. For power factor setting $j$, the DER strategy is defined as $s_{i j}=\left(\gamma_{i j}, c_{i j}\right)$, with $\gamma_{i j}=\left(p f_{i j} \times\right.$ $\left.W_{i}, \sqrt{1-p f_{i j}^{2}} \times W_{i}\right)$. As current pricing mechanisms consider only real power, the cost $c_{i j}$ can be a function of the real power $p f_{i j} \times$ $W_{i}$. Linear or quadratic costs are widely used in literature. Using quadratic costs, $c_{i j}=a p f_{i j}^{2} \times W_{i}^{2}$, where $a$ is a fixed constant.

Using the setting above, we can define two problems as follows:

4.1.1 Real and Reactive Support via Power Plants. In this scenario, the distribution grid operator determines the load that needs to be satisfied for each interval and solves optimal power flow (OPF) equations to determine the real $P$ and reactive $Q$ injections required by each generator in the grid. An example of such OPF equations is shown in [11]. Each generator/plant receives the values $P$ and $Q$ to inject.

The plant, upon receiving the $P$ and $Q$ values solves the problem defined using Equations $1 \mathrm{a}-1 \mathrm{~d}$ and adopts the suggested strategies. Note that in some cases it is possible that the utility requested $P$ and $Q$ values cannot be attained using any combination of strategies even within the threshold of $\delta$. In such cases, the closest possible value will be output.

4.1.2 Real and Reactive Support with Apparent Power Constraint: Some works such as $[18,41]$ schedule the real and reactive power of a number of devices (potentially connected to the same transformer) with a maximum limit on the apparent power that can be injected as opposed to individual targets for real and reactive power. The reason provided by such works is that this constraint ensures that the capacity (power capacity or thermal capacity) of the transformer is not violated. Our problem, by modeling constraints on real and reactive power individually, makes it generalizable. Finer grained capacity constraints targeting real and reactive power individually can be modeled using our approach.

In this scenario, instead of providing real $P$ and reactive $Q$ power targets, we are given an apparent power constraint $W$. Thus, collectively the apparent power output of all the DERs should be within $W$ while the total cost is minimized (or reward maximized). The apparent power constraint can easily be incorporated into our algorithm with the following modified definition:

$$
\min _{X \in \mathcal{X}} C_{X}
$$

subject to:

$$
\begin{aligned}
& \sum_{i \in[N]} \sum_{j \in[M]} p_{i j} X_{i j}=P \\
& \sum_{i \in[N]} \sum_{j \in[M]} q_{i j} X_{i j}=Q \\
& P^{2}+Q^{2} \leq W^{2} \\
& X_{i j} \in\{0,1\}
\end{aligned}
$$

The only difference is the addition of apparent power constraint using Equation $5 \mathrm{~d}$. Note that in this case, $P$ and $Q$ are auxillary variables and are not input parameters. The solution strategy still remains the same. We fill the dynamic programming table $\Phi$ as before. Once $\Phi$ is filled, we find the minimum cost entry from all $\Phi(p:(1+\epsilon) p, q:(1+\epsilon) q, N)$ such that $p^{2}+q^{2} \leq W^{2}$. This will give us with a $(1+\epsilon)^{2}$ bound on the apparent power constraint violation (or $1+\epsilon$ bound if we consider the constraint as $\sqrt{P^{2}+Q^{2}} \leq W$ ). Note that a maximum of $\widehat{W}^{2} p, q$ pairs need to be considered, hence, the algorithm is still polynomial in the input size and $\frac{1}{\epsilon}$.

\subsection{Dynamic PV Curtailment to Increase Penetration}

In this section, we discuss the simpler application of performing PV curtailment dynamically given a curtailment target. This problem is discussed in works such as $[24,27]$ and the load counterpart (i.e. the 


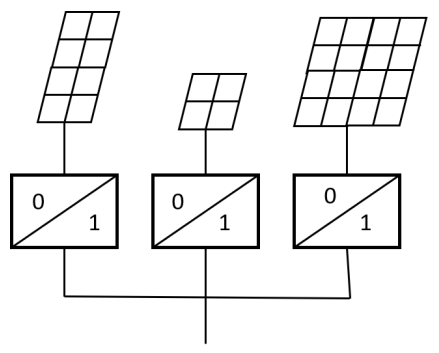

Figure 3: Single PV System: Individual PV modules are connected to micro-inverters which can be turned on/off.

problem in which load is curtailed instead of PV) is widely known as Demand Response [21-23]. We show how a discrete version of this problem can be solved using our approximate DER scheduling algorithm.

As mentioned before, utilities in U.S. typically set a hard limit of $15 \%$ on the amount of renewable energy based DERs such as PVs that can be installed in the distribution system. By enabling dynamic curtailment of PV injection, we can increase the amount of PV that can be installed in the distribution system.

We consider a single DER to be a PV system with multiple inverters, each of which is connected to a bunch of PV modules (Figure 3). We assume that each inverter can be individually turned on/off. This assumption can be implemented using techniques detailed in [12]. Thus, each unique on/off setting for all the inverters in the PV system defines a single strategy. We assume there are multiple such PV systems connected to the grid.

As per the notations defined in Section 3.1, for a PV system $i$, a strategy $j$ is defined as $s_{i j}=\left(\gamma_{\mathbf{i j}}, c_{i j}\right)$. Assuming the power factor of its inverter is $p f_{i}, \gamma_{i j}=\left(p_{i j}, d_{i} \times p_{i j}\right)$, where $d_{i}=\frac{p_{i j} \sqrt{1-p f_{i}}}{p f_{i}}$

Typically, for this problem, the aim is to perform minimum amount of curtailment so that the aggregate real PV injection is within $l$ factor of the load $L$ at any given time. Therefore, only real power values $p_{i j}$ can be considered. Moreover, the costs can simply be set to $c_{i j}=0$ with the problem defined as a feasibility problem as follows:

$$
\min _{X \in \mathcal{X}} 0
$$

subject to:

$$
\begin{aligned}
& \sum_{i \in[N]} \sum_{i \in[N]} p_{i j} X_{i j}=l_{\epsilon} \times L \\
& X_{i j} \in\{0,1\}
\end{aligned}
$$$$
\forall i, j
$$

Here, $l_{\epsilon}=\frac{l}{(1+\epsilon)}$ ensures that the constraint is satisfied even in the worst case. For utilities in U.S., $l=0.15$ i.e. $15 \%$ limit [2]. This problem formulation further simplifies the complexity of the dynamic program by reducing the dimension corresponding to reactive power $q$.

Remark: For a single interval (or multiple independent intervals over a horizon), a better solution can be obtained by our framework by simply switching the costs and real power in our dynamic programming formulation. In other words, instead of creating the dynamic program which takes $i$ and $p$ as input and outputs the minimum cost $c$, creating a dynamic program which takes $i$ and $c$ as input and outputs the minimum $p$ while discretizing costs as mentioned in Section 3.3 will lead to a solution which does not violate the real power constraints. The costs need to be set to $c_{i j}=1$.

\section{THEORETICAL COMPARISON WITH OTHER WORKS}

We compare the theoretical guarantees and runtime complexity of our algorithm against the algorithms developed in [20]. We denote our algorithm as $T$-approx-DER (Section B). We denote the algorithms developed in [20] as $T-\operatorname{CSP}\left[0, \frac{\pi}{2}\right], T-\operatorname{CSP}[0, \pi-\epsilon]$ and 1 - CSP $\left[0, \frac{\pi}{2}\right]$ (We replace $m$ : the notation used in [20] with $T$ to denote time).

It is important to note that the problems $T$-CSP $\left[0, \frac{\pi}{2}\right]$ and $T$ $\operatorname{CSP}[0, \pi-\epsilon]$ have a much more complex setup. $T$-approx-DER assumes that a strategy, when selected, is active for a single interval and strategies across intervals are independent. In contrast, $T$-CSP $\left[0, \frac{\pi}{2}\right]$ and $T-\operatorname{CSP}[0, \pi-\epsilon]$ assume that a selected strategy spans multiple intervals with a pre-defined start and end interval. This difference in the problem setup makes their algorithm more complex than ours.

Hence, to perform a fair comparison, we consider both the problem settings: (i) the strategies across intervals are independent, and (ii) strategies span multiple intervals. We compare how the algorithms perform in both the scenarios. We use $n$ to denote the input size excluding $T$ i.e. $n$ is determined by the number of DERs and the strategies each can follow. The accuracy guarantee is listed as $(\alpha, \beta)$ which means that the objective value will be worse by a factor of $\alpha$ while the constraint will be violated by a factor of $\beta$. The notation $\operatorname{poly}(a)$ means the expression is a polynomial function in $a$.

\subsection{Independent Strategies Across Intervals}

To adapt $T$-CSP $\left[0, \frac{\pi}{2}\right]$ and $T$-CSP $[0, \pi-\epsilon]$ to this problem setting, we need to assume that for each interval, the start and the end interval is the same i.e. if a strategy is available in time $T^{\prime}$, then its start and end intervals are set to be $T^{\prime}$. Under this setting the algorithms $T$-CSP $\left[0, \frac{\pi}{2}\right]$ and $T$-CSP $[0, \pi-\epsilon]$ have PTAS (polynomial in input size, exponential in $\frac{1}{\epsilon}$ ) and FPTAS (fully polynomial in the input size and $\frac{1}{\epsilon}$ ) as solutions assuming $T$ is a constant while our solution $T$-approx-DER has FPTAS even if we consider $T$ to be an input. In Table 2, we compare the runtime and accuracy of the algorithms with $T=1$ as a constant and $T$ as an input parameter.

As evident from the table, when $T$ is an input parameter, only 1 - CSP $\left[0, \frac{\pi}{2}\right]$ performs better than our algorithm in terms of runtime. However, it has a poor accuracy guarantee of $>2$ which is equivalent to choosing $\epsilon=\sqrt{2}-1$ in our algorithm. For constant $T=1$, the algorithm $T-\operatorname{CSP}[0, \pi-\epsilon]$ has similar asymptotic performance as our algorithm and a similar accuracy guarantee.

\subsection{Strategies Span Multiple Intervals}

To adapt $T$-Approx-DER to this setting and develop $T$-Approx-DERspan, we assume that the injection values of each strategy is a vector of size $2 T$ (as opposed to 2 in the current version of $T$-Approx-DER). The $2 T$ values are composed of real and reactive values for each time interval $T$. For the time intervals which are outside the start and the end time of a given strategy, we simply set the values to 0 . Thus, 


\begin{tabular}{|l|l|l|l|l|}
\hline Algorithm & Runtime $T=1$ & Runtime $T$ & Accuracy & Assumptions \\
\hline$T$-CSP $\left[0, \frac{\pi}{2}\right]$ & $O\left((n \epsilon) \frac{1}{\epsilon}\right)$ & $O\left(\frac{n \epsilon}{T} \frac{T}{\epsilon}\right)$ & $(1-\epsilon, 1)$ & $\begin{array}{l}\text { - Does not need the assumption of independent time intervals. } \\
\text { - All DERs should either inject or draw real (or reactive) power. }\end{array}$ \\
\hline$T$-CSP $[0, \pi-\epsilon]$ & $O\left(\operatorname{poly}\left(\frac{1}{\epsilon}, n\right)\right)$ & $O\left(\left(\frac{1}{\epsilon} \operatorname{poly}(n)\right)^{O(T)}\right)$ & $(1,1+o(\epsilon))$ & $\begin{array}{l}\text { • Does not need the assumption of independent time intervals. } \\
\text { Some DERs can inject real (or reactive) power while others } \\
\text { draw real (or reactive) power. }\end{array}$ \\
\hline 1 -CSP $\left[0, \frac{\pi}{2}\right]$ & $O(n \log n)$ & $O(\operatorname{Tn} \log n)$ & $(2-2.82,1)$ & $\bullet$ All DERs should either inject or draw real (or reactive) power. \\
\hline$T$-Approx-DER & $O\left(p o l y\left(\frac{1}{\epsilon}, n\right)\right)$ & $O\left(p o l y\left(\frac{1}{\epsilon}, n, T\right)\right)$ & $(1,1+o(\epsilon))$ & $\begin{array}{l}\text { - Requires the assumption of independent time intervals. } \\
\text { All DERs should either inject or draw real (or reactive) power. }\end{array}$ \\
\hline
\end{tabular}

Table 2: Comparison of runtime complexities of our algorithm with other works. Note that the $T-C S P$ algorithms are developed for a more complicated problem setting - DER strategies spanning multiple time-intervals.

T-Approx-DER-span is a multi-dimensional knapsack problem with dimension $2 T$. The runtime of this algorithm will be polynomial in $\frac{1}{\epsilon}$ and $N^{T}, N$ being the number of DERs, which is similar to the complexity obtained by $T-\operatorname{CSP}[0, \pi-\epsilon]$ and significantly better than $T$-CSP $\left[0, \frac{\pi}{2}\right]$. However, it is important to note that $T$-CSP $[0, \pi-\epsilon]$ is a more generalized version that our problem. We only consider the absolute values of the real and reactive powers of the DER strategies, thus restricting our problem to scenarios where all the DERs are either injecting or drawing real power and all DERs are either injecting or drawing reactive power. $T-\operatorname{CSP}[0, \pi-\epsilon]$ handles the cases where some DERs inject real power (or reactive) and others draw real power (or reactive).

\section{EXPERIMENTAL RESULTS}

We validate the theoretical guarantees provided by the ApproxDER algorithm by quantitatively evaluating over the applications Real and Reactive Support Using Smart Inverters and Dynamic PV Curtailment to Increase Penetration as listed in Section 4. We implement an unoptimized python program (available at [5]) on a PC with i5 processor $(2.5 \mathrm{GHz})$ and $8 \mathrm{~GB}$ of memory. We evaluate the scalability of the applications with respect to varying number of DERs and the accuracy parameter $\frac{1}{\epsilon}$ and the optimality guarantee of the algorithm.

\subsection{Datasets}

The DERs that we consider for evaluation in this work are PV systems. We use PVLib-python [14], which is a python port of PVLib MATLAB developed by Sandia National Labs [33] to simulate the PV system output. PVlib takes as input the PV system configuration: type of modules, number of modules, series/parallel layout of the modules; type of smart inverter, location and time period, and produces the AC output for each time period for the PV system at the specified location. We set the location to be as Los Angeles, United States. We select an inverter with a maximum output power of $3 \mathrm{MW}$. We vary the number of modules from 1 to 6 and vary the series/parallel layout of the modules to simulate different kinds of DERs. Using two types of modules, we simulate data for a total of 20 DERs for a randomly selected day of 1st April, 2017.

For the application: Real and Reactive Support, we use 4 power factor settings $[0.97,0.98,0.99,1.0]$ for each smart inverters. Thus, each PV system is associated with 5 settings: 4 corresponding to the power factors and one strategy where the PV system is turned off. We vary the values of the real $P$ and reactive $Q$ power target values to perform detailed experiments.

For the application: Dynamic PV Curtailment, we fix the power factor setting to 1.0, and assume each micro-inverter has an on/off setting. Thus, the maximum number of strategies for any PV system is 6 - when 6 modules are connected in parallel via inverter to the grid. We vary the value of load in the feeder $L$ to perform detailed experiments.

\subsection{Scalability Analysis}

To perform scalability analysis, we simply duplicate the output of 20 DERs to create a dataset for 100 DERs. We assign $P$ and $Q$ to be the maximum possible value that can be achieved using the DERs.

For the application: real and reactive support, we vary the number of DERs $N$ from 10 to 50. Figure 4 (a) shows the runtime for various values of $\epsilon$. The runtime increases from less than a second for $N=10$ to 639 seconds for $N=50, \epsilon=0.05$ (5\%) and 182 seconds for $N=50, \epsilon=0.5$ (50\%). As evident from the figure, the increase in runtime is of polynomial complexity. Moreover, the higher the accuracy (lower $\epsilon$ ), the lower is the scalability. In Section C.1, we discuss a few techniques which can further improve the scalability of our algorithm.

For the application: dynamic PV curtailment, as only one dimension (corresponding to real power) is needed in the dynamic programming table, the runtimes are significantly better. We vary the number of DERs $N$ from 10 to 100 . Figure 4 (b) shows the runtime for various values of $\epsilon$. The maximum runtime in this case is around 18 seconds for $N=100, \epsilon=0.01(1 \%)$. 


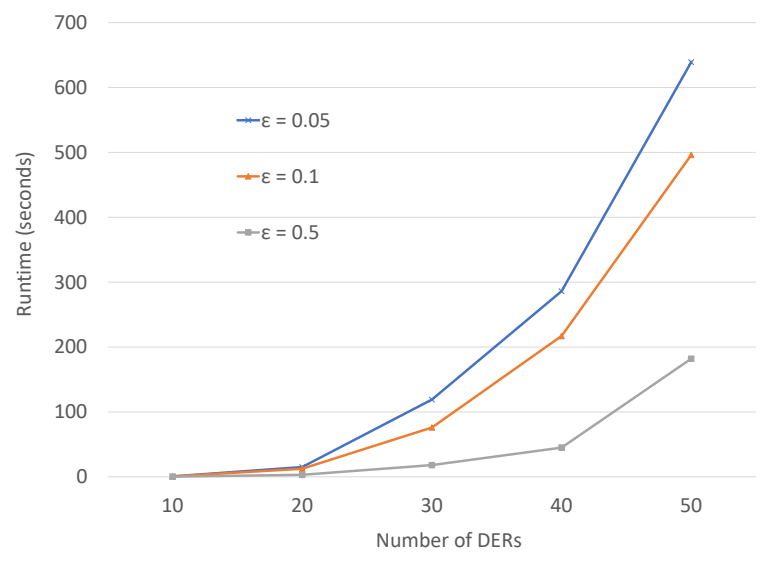

(a) Application: Real and Reactive Support

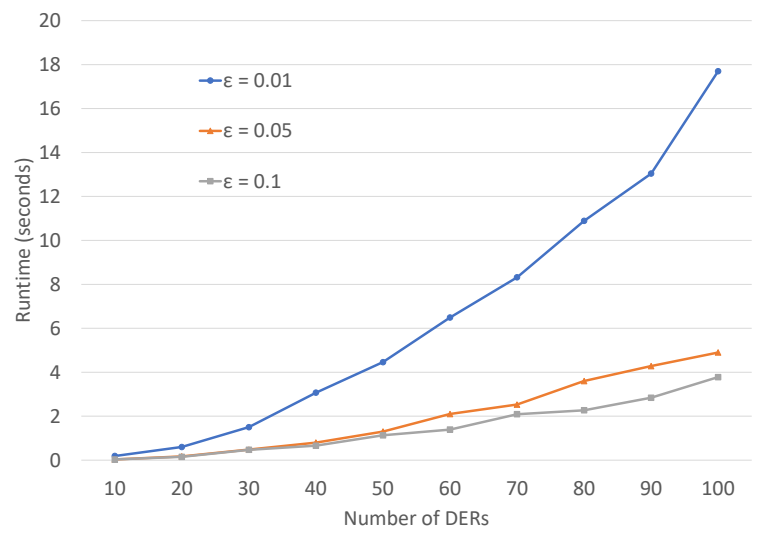

(b) Application: Dynamic PV Curtailment

Figure 4: Runtime versus Number of DERs for both the applications using Approx-DER scheduling algorithm

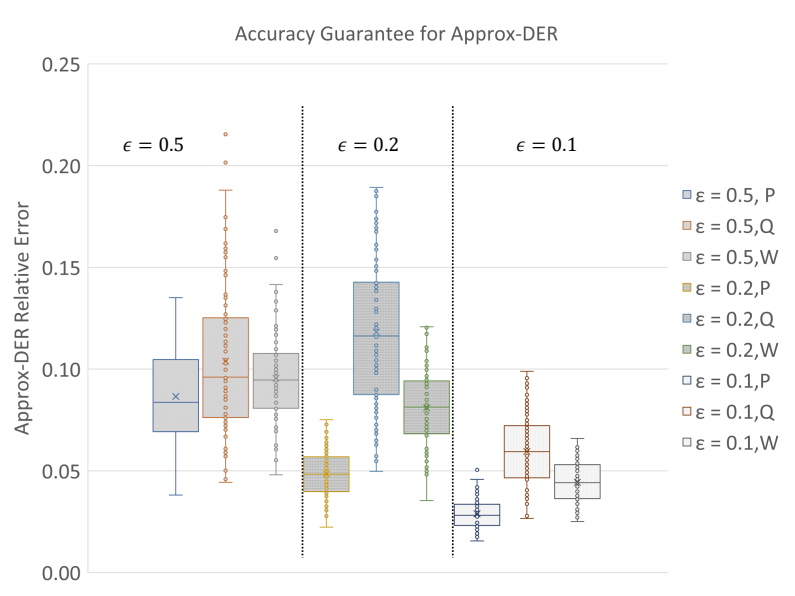

Figure 5: Distribution of Errors incurred by Approx-DER for real $P$, reactive $Q$ and Apparent $W$ power for various values of $\epsilon$. (For clarity, the markers on $\mathrm{x}$ axis are presented as legends instead.)

\subsection{Optimality}

To evaluate the accuracy guarantee provided by Approx-DER on real power $P$, reactive power $Q$ and apparent power $W$ constraints, we vary the value of $P$ from $\frac{P_{\max }}{8}$ to $P_{\max }$ and the value of $Q$ from $\frac{Q_{\max }}{8}$ to $Q_{\max }$ and calculate the relative error i.e. $\frac{P_{a}-P}{P}, \frac{Q_{a}-Q}{Q}$ and $\frac{W_{a}-W}{W}$, where $P_{a}, Q_{a}, W_{a}$ are the values achieved using ApproxDER. The results for values of $\epsilon=0.5,0.2,0.1$ are shown in Figure 5.

As evident from the figure, the maximum error incurred for each value of $\epsilon$ is within the theoretical guarantee provided. For higher accuracy (lower $\epsilon$ ), the errors incurred are closer to the guarantee. The median error (horizon line inside the boxes) incurred for $\epsilon=0.5$ is around 0.1 , for $\epsilon=0.2$ varies from 0.05 for real power constraint to 0.12 for reactive power constraint and for $\epsilon=0.1$ it varies from 0.03 to 0.07 .

We also compare the cost of the solutions obtained by ApproxDER with the optimal solutions. The optimal solutions are obtained by running a dynamic program which does not discretize and considers all possible values of real and reactive injections to calculate the cost. We again calculate the relative error defined as $\frac{C_{a}-C}{C}$ where $C$ is the cost of the optimal solution and $C_{a}$ is the the cost of the solution output by Approx-DER. The results for values of $\epsilon=0.5,0.2,0.1$ are shown in Figure 6. Note that the relative errors shown in the figure are negative as the cost of Approx-DER is less than or equal to the cost of the optimal solutions due to its violation of constraints. Moreover, as evident from the figure, the relative errors increase with increase in the value of $\epsilon$ which allows for a wider range of constraint violation.

\section{ACKNOWLEDGEMENTS}

We would like to acknowledge the shepherd Sid Chi-Kin Chau and the anonymous reviewers for their useful feedback. This work has been funded by the U.S. Department of Energy under award number DE-EE0008003 and the National Science Foundation under award number CNS-1637372.

\section{CONCLUSION}

Dramatic technological and algorithmic advances are required to ensure the rapid pace of increase in the penetration of renewable energy based DERs is sustained. The ability to efficiently schedule DERs, several of which exhibit discrete strategy space, is one such algorithmic advancement that is needed.

We provide the first near optimal fully polynomial approximation algorithm for efficiently scheduling DERs whose feasible set of injections forms a discrete space. We provide theoretical guarantees for runtime and near optimality and validate these guarantees by performing detailed experiments. We also illustrate the applicability of our algorithms by discussing two real world applications. 


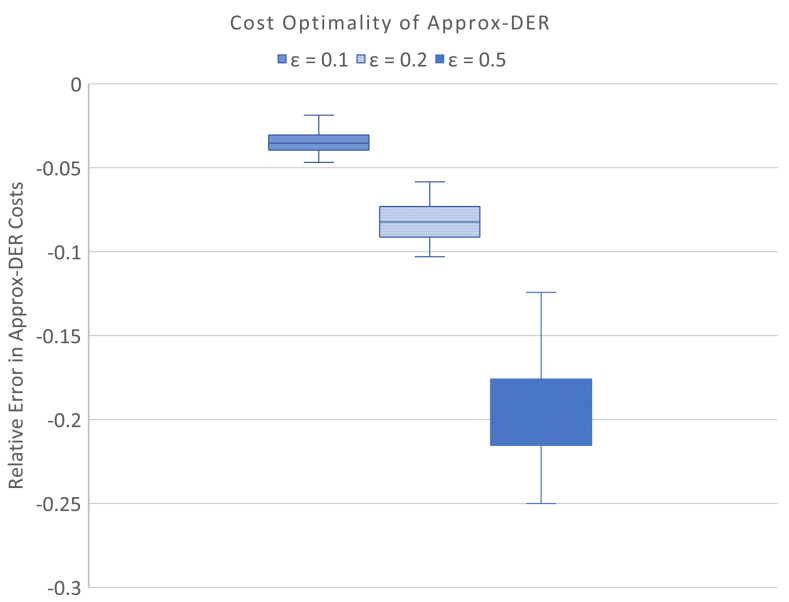

Figure 6: Distribution of Errors incurred in the cost of solutions obtained by Approx-DER for various values of $\epsilon$. (For clarity, the markers on $x$ axis are presented as legends instead.)

\section{REFERENCES}

[1] 2012. Common Functions for Smart Inverter. Online: http://www.epri.com/abstracts/Pages/ProductAbstract.aspx?ProductId=1026809. http://www.epri.com/abstracts/Pages/ProductAbstract.aspx?ProductId= 1026809

[2] 2017. Hosting Capacity: Using Increased Transparency of Grid Constraints to Accelerate Interconnection Processes. Online:https://www.seia.org/sites/default/files/2017-09/SEIA-GridModSeries-3_2017-Sep-FINAL.pdf. https://www.seia.org/sites/default/files/2017-09/ SEIA-GridMod-Series-3_2017-Sep-FINAL.pdf

[3] 2018. Hawaii State Energy Office. Online. https://energy.hawaii.gov/ renewable-energy

[4] 2018. SB-100 California Renewables Portfolio Standard Program: emissions of greenhouse gases. Online. https://leginfo.legislature.ca.gov/faces/billNavClient. xhtml?bill_id=201720180SB100

[5] 2019. Approx-DER CodeBase. Online. https://github.com/sanmukh/approxDER

[6] Soroush Alamdari, Therese Biedl, Timothy M Chan, Elyot Grant, Krishnam Raju Jampani, Srinivasan Keshav, Anna Lubiw, and Vinayak Pathak. 2013. Smart-grid electricity allocation via strip packing with slicing. In Workshop on Algorithms and Data Structures. Springer, 25-36.

[7] Karthikeyan Balasubramaniam, Parimal Saraf, Ramtin Hadidi, and Elham B Makram. 2016. Energy management system for enhanced resiliency of microgrids during islanded operation. Electric Power Systems Research 137 (2016), 133-141.

[8] Vito Calderaro, Vincenzo Galdi, Francesco Lamberti, and Antonio Piccolo. 2015. A smart strategy for voltage control ancillary service in distribution networks. IEEE Transactions on Power Systems 30, 1 (2015), 494-502.

[9] Sid Chi-Kin Chau, Khaled Elbassioni, Majid Khonji, et al. 2018. Combinatorial Optimization of Alternating Current Electric Power Systems. Foundations and Trends ${ }^{\circledR}$ in Electric Energy Systems 3, 1-2 (2018), 1-139.

[10] Thomas Erlebach, Hans Kellerer, and Ulrich Pferschy. 2002. Approximating multiobjective knapsack problems. Management Science 48, 12 (2002), 16031612.

[11] Masoud Farivar and Steven H Low. 2013. Branch flow model: Relaxations and convexificationâĂŤPart I. IEEE Transactions on Power Systems 28, 3 (2013), 25542564.

[12] Ognjen Gagrica, Phuong H Nguyen, Wil L Kling, and Tadeusz Uhl. 2015. Microinverter curtailment strategy for increasing photovoltaic penetration in low-voltage networks. IEEE Transactions on Sustainable Energy 6, 2 (2015), 369-379.

[13] Bezalel Gavish and Hasan Pirkul. 1985. Efficient algorithms for solving multiconstraint zero-one knapsack problems to optimality. Mathematical programming 31, 1 (1985), 78-105.

[14] William F Holmgren, Clifford W Hansen, and Mark A Mikofski. 2018. pvlib python: a python package for modeling solar energy systems. The fournal of Open Source Software 3 (2018), 884.

[15] Shaun Howell, Yacine Rezgui, Jean-Laurent Hippolyte, Bejay Jayan, and Haijiang Li. 2017. Towards the next generation of smart grids: Semantic and holonic multiagent management of distributed energy resources. Renewable and Sustainable
Energy Reviews 77 (2017), 193-214.

[16] Srinivas Katipamula and Ning Lu. 2006. Evaluation of Residential HVAC Control Strategies for Demand Response Programs. ASHRAE transactions 112, 1 (2006).

[17] Majid Khonji, Chi-Kin Chau, and Khaled Elbassion. 2017. Combinatorial Optimization of AC Optimal Power Flow in Radial Distribution Networks. arXiv preprint arXiv:1709.08431 (2017).

[18] Majid Khonji, Sid Chi-Kin Chau, and Khaled Elbassioni. 2018. Approximation scheduling algorithms for electric vehicle charging with discrete charging options. In Proceedings of the Ninth International Conference on Future Energy Systems. ACM, 579-585.

[19] Majid Khonji, Sid Chi-Kin Chau, and Khaled Elbassioni. 2018. Challenges in Scheduling Electric Vehicle Charging with Discrete Charging Rates in AC Power Networks. In Proceedings of the Ninth International Conference on Future Energy Systems. ACM, 183-186.

[20] Majid Khonji, Areg Karapetyan, Khaled Elbassioni, and Sid Chi-Kin Chau. 2018. Complex-demand scheduling problem with application in smart grid. Theoretical Computer Science (2018).

[21] Sanmukh R Kuppannagari, Rajgopal Kannan, Charalampos Chelmis, and Viktor K Prasanna. 2016. Implementation of Learning-Based Dynamic Demand Response on a Campus Micro-Grid. In The 25th International Joint Conference on Artificial Intelligence.

[22] Sanmukh R Kuppannagari, Rajgopal Kannan, Charalampos Chelmis, Arash S Tehrani, and Viktor K Prasanna. 2016. Optimal Customer Targeting for Sustainable Demand Response in Smart Grids. Procedia Computer Science 80 (2016), 324-334.

[23] Sanmukh R Kuppannagari, Rajgopal Kannan, and Viktor K Prasanna. 2015. An ILP based Algorithm for Optimal Customer Selection for Demand Response in SmartGrids. In The 2015 International Conference on Computational Science and Computational Intelligence (CSCI).

[24] Sanmukh R Kuppannagari, Rajgopal Kannan, and Viktor K Prasanna. 2017. Optimal net-load balancing in smart grids with high PV penetration. In Proceedings of the 4th ACM International Conference on Systems for Energy-Efficient Built Environments. ACM, 27.

[25] Sanmukh R Kuppannagari, Rajgopal Kannan, and Viktor K Prasanna. 2018. NOLESS: Near optimal curtailment strategy selection for net load balancing in micro grids. In 2018 IEEE Power \& Energy Society Innovative Smart Grid Technologies Conference (ISGT). IEEE, 1-5.

[26] Sanmukh R Kuppannagari, Rajgopal Kannan, and Viktor K Prasanna. 2018. Optimal Discrete Net-Load Balancing in Smart Grids with High PV Penetration. ACM Transactions on Sensor Networks (TOSN) 14, 3-4 (2018), 24.

[27] Stephen Lee, Srinivasan Iyengar, David Irwin, and Prashant Shenoy. 2017. Distributed Rate Control for Smart Solar Arrays. In Proceedings of the Eighth International Conference on Future Energy Systems. ACM, 34-44.

[28] Xiaodong Liang. 2017. Emerging power quality challenges due to integration of renewable energy sources. IEEE Transactions on Industry Applications 53, 2 (2017), 855-866.

[29] Milana Plećaš, Han Xu, and Ivana Kockar. 2017. Integration of energy storage to improve utilisation of distribution networks with active network management schemes. CIRED-Open Access Proceedings fournal 2017, 1 (2017), 1845-1848.

[30] Anshu Ranjan, Pramod Khargonekar, and Sartaj Sahni. 2014. Offline preemptive scheduling of power demands to minimize peak power in smart grids. In Computers and Communication (ISCC), 2014 IEEE Symposium on. IEEE, 1-6.

[31] Alireza Rezvani, Majid Gandomkar, Maziar Izadbakhsh, and Abdollah Ahmadi. 2015. Environmental/economic scheduling of a micro-grid with renewable energy resources. Fournal of cleaner production 87 (2015), 216-226.

[32] Ward Romeijnders, Leen Stougie, and Maarten H van der Vlerk. 2014. Approximation in two-stage stochastic integer programming. Surveys in Operations Research and Management Science 19, 1 (2014), 17-33.

[33] Joshua S Stein, William F Holmgren, Jessica Forbess, and Clifford W Hansen. 2016. PVLIB: Open source photovoltaic performance modeling functions for Matlab and python. In 2016 IEEE 43rd Photovoltaic Specialists Conference (PVSC). IEEE, 3425-3430.

[34] Shaojie Tang, Qiuyuan Huang, Xiang-Yang Li, and Dapeng Wu. 2013. Smoothing the energy consumption: Peak demand reduction in smart grid. In INFOCOM, 2013 Proceedings IEEE. IEEE, 1133-1141.

[35] Europäische Union. 2009. Directive 2009/28/EC of the European Parliament and of the Council of 23 April 2009 on the promotion of the use of energy from renewable sources and amending and subsequently repealing Directives 2001/77/EC and 2003/30/EC. Official fournal of the European Union 5 (2009), 2009.

[36] John S Vardakas, Nizar Zorba, and Christos V Verikoukis. 2015. A survey on demand response programs in smart grids: Pricing methods and optimization algorithms. IEEE Communications Surveys \& Tutorials 17, 1 (2015), 152-178.

[37] Rajiv K Varma and Ehsan M Siavashi. 2018. PV-STATCOM-A New Smart Inverter for Voltage Control in Distribution Systems. IEEE Transactions on Sustainable Energy (2018).

[38] Qinglong Wang, Xue Liu, Jian Du, and Fanxin Kong. 2016. Smart charging for electric vehicles: A survey from the algorithmic perspective. IEEE Communications Surveys \& Tutorials 18, 2 (2016), 1500-1517. 
[39] Gerhard J Woeginger. 2000. When does a dynamic programming formulation guarantee the existence of a fully polynomial time approximation scheme (FPTAS)? INFORMS fournal on Computing 12, 1 (2000), 57-74.

[40] Sean Yaw, Brendan Mumey, Erin McDonald, and Jennifer Lemke. 2014. Peak demand scheduling in the smart grid. In Smart Grid Communications (SmartGridComm), 2014 IEEE International Conference on. IEEE, 770-775.

[41] Ao Zhang, Bo Sun, Tian Liu, Xiaoqi Tan, Su Wang, and Danny HK Tsang. 2018 Joint voltage and frequency regulation by EV charging scheduling in the distribution network. In 2018 IEEE Power \& Energy Society Innovative Smart Grid Technologies Conference (ISGT). IEEE, 1-5.

\section{APPENDICES}

\section{A PROOF OF THEOREM 3.1}

Correctness: We will prove the correctness of the algorithm in two steps. In step 1, we will prove that the dynamic program runs correctly to fill $\Phi$ and step 2, we will prove that, if the dynamic program runs on actual values instead of the partition indices and an actual value can be achieved, then this information is not missed in dynamic program run on partition indices.

We will prove step 1 by induction. Essentially we need to prove that if $\Phi(\widehat{p}, \widehat{q}, i)<\infty$ iff it is possible to achieve $\widehat{p}, \widehat{q}$ using DERs 1 through $i$ at cost $\Phi(\widehat{p}, \widehat{q}, i)$ with this cost being the minimum. For $i=1$, it is trivially true, as we will chose the strategy $s_{1 j}$ of minimum cost which achieves $\widehat{p}, \widehat{q}$. Let, the inductive hypothesis be true for $i=k$. So, $\Phi(\widehat{p}, \widehat{q}, k)$ is the minimum cost of achieving $\widehat{p}, \widehat{q}$ using 1 through $k$ nodes. Now, consider $\Phi\left(\widehat{p}^{\prime}, \widehat{q}^{\prime}, k+1\right)$, as per Equation $2, \Phi\left(\widehat{p}^{\prime}, \widehat{q}^{\prime}, k+1\right)$ is the minimizer over all $\widehat{p}, \widehat{q}$ which when added with the output of some DER strategy $s_{(k+1) j}$ achieves $\widehat{p}^{\prime}, \widehat{q}^{\prime}$. As $\Phi(\widehat{p}, \widehat{q}, k)$ was minimum cost, $\Phi\left(\widehat{p}^{\prime}, \widehat{q}^{\prime}, k+1\right)$ is also a minimizer.

To prove step 2, we show that if using $k$ nodes, an actual value of $p_{a}^{k}=\sum_{i=1}^{k} p_{i}, q_{a}^{k}=\sum_{i=1}^{k} q_{i}$ can be achieved, then $\widehat{p}_{a}^{k} \leq \sum_{i=1}^{k} p_{i}<$ $(1+\epsilon)^{\frac{k}{N}} \widehat{p}_{a}^{k}$ and $\widehat{q}_{a}^{k} \leq \sum_{i=1}^{k} q_{i}<(1+\epsilon)^{\frac{k}{N}} \widehat{q}_{a}^{k}$ and some entries $\Phi\left(\hat{p}_{a}^{k}:(1+\epsilon)^{\frac{k}{N}} \widehat{p}_{a}^{k}, \widehat{q}_{a}^{k}:(1+\epsilon)^{\frac{k}{N}} \widehat{q}_{a}^{k}, k\right)<\infty$. We again use induction. For $k=1$, if $p_{1}=p_{a}^{1} \in \widehat{p}_{a}^{1}, q_{1}=q_{a}^{1} \in \widehat{q}_{a}^{1}$ can be achieved using some strategy $s_{1 j}$, then the entry $\Phi\left(\widehat{p}_{a}^{1}, \widehat{q}_{a}^{1}, 1\right)=c_{1 j}<\infty$ and $\widehat{p}_{a}^{1} \leq p_{1}<(1+\epsilon)^{\frac{1}{N}} \widehat{p}_{a}^{1}$ and $\widehat{q}_{a}^{1} \leq q_{1}<(1+\epsilon)^{\frac{1}{N}} \widehat{q}_{a}^{1}$. Let the inductive hypothesis be true for $k^{\prime}$ i.e. if a value of $p_{a}^{k^{\prime}}=$ $\sum_{i=1}^{k^{\prime}} p_{i}, q_{a}^{k^{\prime}}=\sum_{i=1}^{k^{\prime}} q_{i}$ can be achieved, then $\widehat{p}_{a}^{k^{\prime}} \leq \sum_{i=1}^{k^{\prime}} p_{i}<$ $(1+\epsilon)^{\frac{k}{N}} \widehat{p}_{a}^{k^{\prime}}$ and $\widehat{q}_{a}^{k^{\prime}} \leq \sum_{i=1}^{k} q_{i}<(1+\epsilon)^{\frac{k}{N}} \widehat{q}_{a}^{k^{\prime}}$ and some entries $\Phi\left(\widehat{p}_{a}^{k^{\prime}}:(1+\epsilon)^{\frac{k}{N}} \widehat{p}_{a}^{k^{\prime}}, \widehat{q}_{a}^{k^{\prime}}:(1+\epsilon)^{\frac{k}{N}} \widehat{q}_{a}^{k^{\prime}}, k^{\prime}\right)<\infty$. For $k^{\prime}+1$, as per the definition of the dynamic program, at least the entry where $\widehat{p}_{a}^{k^{\prime}}+p_{k^{\prime}+1}$ and $\widehat{q}_{k}+q_{k^{\prime}+1}$ falls will have a cost of $<\infty$. We just need to show that $\widehat{p}_{a}^{k^{\prime}+1} \leq p_{a}^{k^{\prime}+1}=\sum_{i=1}^{k^{\prime}+1} p_{i}<(1+\epsilon)^{\frac{k^{\prime}}{N}} \widehat{p}_{a}^{k^{\prime}+1}$ and $\widehat{q}_{a}^{k^{\prime}+1} \leq q_{a}^{k^{\prime}+1}=\sum_{i=1}^{k^{\prime}+1} q_{i}<(1+\epsilon)^{\frac{k^{\prime}}{N}} \widehat{q}_{a}^{k^{\prime}+1}$.

We know the following: (1) $\widehat{p}_{a}^{k^{\prime}+1} \leq \widehat{p}_{a}^{k^{\prime}}+p_{k^{\prime}+1}<(1+\epsilon)^{\frac{1}{N}} \widehat{p}_{a}^{k^{\prime}+1}$ and (2) $\sum_{i=1}^{k^{\prime}} p_{i}<(1+\epsilon)^{\frac{k^{\prime}}{N}} \widehat{p}_{a}^{k^{\prime}}$. So, $\sum_{i=1}^{k^{\prime}+1} p_{i}<(1+\epsilon)^{\frac{k^{\prime}}{N}} \widehat{p}_{a}^{k^{\prime}}+p_{k^{\prime}+1}<$ $(1+\epsilon)^{\frac{k^{\prime}}{N}}\left(\widehat{p}_{a}^{k^{\prime}}+p_{k^{\prime}+1}\right)<(1+\epsilon)^{\frac{k^{\prime}}{N}}(1+\epsilon)^{\frac{1}{N}} \widehat{p}_{a}^{k^{\prime}+1}<(1+\epsilon)^{\frac{k^{\prime}+1}{N}} \widehat{p}_{a}^{k^{\prime}+1}$ as $(1+\epsilon)^{\frac{k^{\prime}}{N}}>1$, when $\epsilon, k^{\prime}>0$. We can prove the result for $q$ similarly.

Runtime: The dynamic programming table has $k_{p} \times k_{q} \times N$ entries. Each entry requires $M$ time to fill. Hence, the total runtime is $k_{p} \times k_{q} \times N \times M=O\left(N^{3} M \log _{1+\epsilon}\left(\frac{P}{P_{\text {min }}}\right) \log _{1+\epsilon}\left(\frac{Q}{Q_{\text {min }}}\right)\right)$. We assume $\log \left(\frac{P}{P_{\text {min }}}\right)$ and $\log \left(\frac{Q}{Q_{\text {min }}}\right)$ is polynomial in the input size. In order words, $\frac{P}{P_{\min }}$ and $\frac{P}{P_{\text {min }}}$ can be exponential in input size but may not be doubly exponential. Moreover, $\log (1+\epsilon) \sim \epsilon-\frac{\epsilon^{2}}{2}+$ $\frac{\epsilon^{3}}{3} \ldots$, so, $\frac{1}{\log (1+\epsilon)} \sim \operatorname{poly}\left(\frac{1}{\epsilon}\right)$. Here poly $(a)$ implies a polynomial in function in $a$. Hence, the runtime complexity of our algorithm is $O\left(\operatorname{poly}\left(\frac{1}{\epsilon}, N, M\right)\right)$.

Approximation Guarantee: Our algorithm outputs the minimum cost solution from the entries $\Phi(\widehat{P}:(1+\epsilon) \widehat{P}, \widehat{Q}:(1+\epsilon) \widehat{Q}, N)$. For $k=N$, DERs as per the proof above: $\widehat{P} \leq P<(1+\epsilon) \widehat{P}$ and $\widehat{Q} \leq Q<(1+\epsilon) \widehat{Q}$, so clearly the solution output by the algorithms is less than or equal to the actual minimum cost solution. Additionally, if $P=\widehat{P}$ then the achieved value $P_{a}<(1+\epsilon) \widehat{P}=(1+\epsilon) P$. Similarly, if $P \rightarrow(1+\epsilon) \widehat{P}$, then $P<(1+\epsilon) \widehat{P}<(1+\epsilon) P_{a}$. Similarly, we can show the result for $Q$.

Note that the rounding scheme for approximation used in our work is similar to the one used in [10].

\section{B SCHEDULING DERS ACROSS MULTIPLE INTERVALS}

In this section, we discuss how Approx-DER can be used (repeatedly) to schedule DERs across multiple intervals with some interdependencies in the constraints across the intervals. Consider the following problem:

$$
\min _{X(t) \in \mathcal{X}(t)} C_{X}(t)
$$

subject to:

$$
\begin{array}{ll}
\sum_{i \in[N]} \sum_{j \in[M]} p(t)_{i j} X(t)_{i j}=P(t) \quad & \forall t \\
\sum_{i \in[N]} \sum_{j \in[M]} q(t)_{i j} X(t)_{i j}=Q(t) & \forall t \\
P(t)_{\min } \leq P(t) \leq P(t)_{\max } & \\
Q(t)_{\min } \leq Q(t) \leq Q(t)_{\max } & \\
\sum_{t \in[T]} P(t)=P & \\
\sum_{t \in[T]} Q(t)=Q & \\
X(t)_{i j} \in\{0,1\} & \forall i, j, t
\end{array}
$$

Here, $p(t)_{i j}, q(t)_{i j}, x(t)_{i j}$ denote the respective values at time $t \in$ $[T]$. In each interval $t$, we assume that the achieved real and reactive values have some upper and lower limits. The global constraints creating the interdependency are Equations $7 \mathrm{f}$ and $7 \mathrm{~g}$, with the constraint being that the sum of real and reactive values of all DERs across the scheduling horizon should sum up to $P$ and $Q$ respectively.

We briefly outline how this problem can be solved using ApproxDER scheduling algorithm. For each interval $t$, using Approx-DER, we will fill the $\Phi^{t}$ table. Now, we will create a set $s_{t}=\left\{\Phi^{t}(\widehat{p}, \widehat{q}, N)\right.$ $\left.\mid \widehat{P}(t)_{\text {min }} \leq \widehat{p}<(1+\epsilon) \widehat{P}(t)_{\text {max }} \& \& \widehat{Q}(t)_{\text {min }} \leq \widehat{q}<(1+\epsilon) \widehat{Q}(t)_{\text {max }}\right\}, \forall t$ On the sets $s_{t}$, we will again call Approx-DER with each time interval $t$ considered as a single DER and its corresponding strategies defined as the entries of set $s_{t}$ to obtain the final solution.

It is easy to show that the runtime is still polynomial in $N, M, T$ and $\frac{1}{\epsilon}$. Moreover, the approximation guarantee on achieving the 
final $P$ and $Q$ values is $(1+\epsilon)^{2}$. Note that this is just one example of a multi-interval problem that can be solved by our algorithm. The simplicity of our algorithm allows several variants to be defined and solved easily without significantly losing the runtime and approximation guarantees.

\section{LIMITATIONS}

In this section, we list a few limitations of our algorithm and potential approaches that can be followed to address them. We will perform rigorous analyses to evaluate their effectiveness in future works.

\section{C.1 Scalability}

The runtime of Approx-DER Scheduling algorithm, $O\left(N^{3} M n_{p} n_{q}\right)$ with $n_{p}=\log _{1+\epsilon}\left(\frac{P_{\max }}{P_{\min }}\right)$ and $n_{q}=\log _{1+\epsilon}\left(\frac{Q_{\max }}{Q_{\min }}\right)$, while fully polynomial is still very large. Most significantly the scalability in the number of DERs $N$ is limited.

However, several techniques can be used to improve the runtime. All the entries $\Phi(:,:, i)$ corresponding to DER $i$ can be filled in parallel. (We use the notation : to denote all entries in a given dimension). Thus our algorithm is highly parallelizable which can lead to significant performance improvements. Moreover, as the runtime is limited by the number of DERs $N$, a simple partitioning technique can be used to improve the runtime. The technique is as follows: Create $l$ partitions of the DERs with each partition consisting of $\frac{N}{l}$ nodes. Create dynamic programming tables $\Phi_{l_{i}}$ for each partition $l_{i} \in l$ using Approx-DER scheduling algorithm. Create sets $s_{l_{i}}=\Phi_{l_{i}}\left(:,:, \frac{N}{l}\right)$. Again run Approx-DER scheduling algorithm with each partition $l_{i}$ as a DER and the elements of set $s_{l_{i}}$ as the corresponding strategies to obtain the final solution. This approach will result in an approximation guarantee of $(1+\epsilon)^{2}$ with a runtime of $\max \left\{O\left(l\left(\frac{N}{l}\right)^{3} M n_{p} n_{q}\right), O\left(l^{3} n_{p}^{2} n_{q}^{2}\right)\right\}$. Assuming $n_{p}, n_{q}=$ $N$, this leads to a lower runtime when $l^{3}<<N M$.

In this work, we limit our efforts to prove the existence of an approximate DER scheduling algorithm which is fully polynomial in the input size and $\frac{1}{\epsilon}$. We defer the implementation of a scalable real-time DER scheduling framework for future works.

\section{C.2 Fairness}

The objective of Approx-DER scheduling algorithms is to minimize cost while achieving the real and reactive power target. Our algorithm does not explicitly consider fairness. In other words, it is possible that some DERs are forced to adopt strategies that have significantly higher injection value than the others.

One approach to address this issue is to properly assign the costs. As mentioned in [27], using logarithm as reward function (negative of cost function) ensures proportional fairness in the scenario where the injections are continuous. So, for a strategy $s_{i j}$, assigning a cost function of $\log \left(p_{i j}\right)$ should provide balanced results. However, additional research is required to develop a fair version of approx-DER which is beyond the scope of this work.

\section{C.3 Prediction Uncertainty}

In a real world deployment scenario, the input real and reactive injection values will be provided by prediction models. As ApproxDER is a deterministic algorithm, it cannot handle randomness due to prediction errors from these models. This could potentially lead to higher errors than the theoretical guarantee.

There are several methods to address this limitation. One method is to run Approx-DER as close to real time as possible with the most updated injection data to reduce errors from the prediction models. Another method is to choose $\epsilon$ conservatively to account for the worst case prediction errors.

However, the most promising approach is to formulate the problem as a two stage recourse model [32]. Under this model, the decision making takes place in two stages: i) In stage one, the decisions minimizing cost and satisfying constraints are made to dispatch the DERs, and in ii) any imbalances due to the decisions made in stage 1 are mitigated, potentially at a higher cost, using devices such as storage. The techniques developed in [32], along with the simplicity of our algorithm will ensure that with high probability a theoretical guarantee of $1+O(\epsilon)$ can be provided. We defer the detailed analysis for a future work.

\section{C.4 Discrete Optimal Power Flow}

In Section 4.1, we discuss how Approx-DER can be used to provide real and reactive support by a large power plant to the grid. We assume that the utility solves an optimal power flow problem to determine the $P$ and $Q$ values to be injected by the power plant. However, it is possible the utility determined $P$ and $Q$ values are not achievable by the plant as it is not an element of the discrete space of the feasible injections of the plant.

To avoid such scenarios, we need efficient algorithms to solve optimal power flow equations which can consider the power injections to be both continuous values as well as discrete values. As shown in this work [19] this is a challenging problem and the current best result is a PTAS developed in $[9,17]$. 